\title{
Radiation Induced Grafting of Acrylate onto Waste Rubber: The Effect of Monomer Type
}

\author{
Siti Salwa M. Shirajuddin ${ }^{1, *}$, Che Mohd Ruzaidi Ghazali ${ }^{2,3}$, Chantara Thevy Ratnam ${ }^{1}$, \\ Kamarudin Husin ${ }^{2}$, Nor Azwin Shukri ${ }^{1}$, and Nur Azillah Fatimah Othman ${ }^{1}$ \\ ${ }^{1}$ Agensi Nuklear Malaysia, Bangi 43000 Kajang, Selangor, Malaysia \\ ${ }^{2}$ Universiti Malaysia Perlis, 01000 Kangar, Perlis, Malaysia \\ ${ }^{3}$ School of Fundamental Science, Universiti Malaysia Terengganu, 21030 Kuala Terengganu, \\ Terengganu
}

\begin{abstract}
The effect of three different acrylate group monomers, namely n-butyl acrylate, methacrylic acid and tripropylene glycol diacrylate of radiation induced grafting onto waste rubber was studied. The electron beam accelerator operated at voltage of $2 \mathrm{MeV}$ was used to irradiate the waste rubber at $10 \mathrm{kGy}$ and $100 \mathrm{kGy}$ absorbed radiation dose, respectively. The formation of grafting was observed from the increase in the grafting yield and confirmed by Transformed Infra-Red Spectroscopy results. According to the result obtained, only tripropylene glycol diacrylate was selected to graft onto waste rubber. The carbonyl bond from acrylate groups was seen at $1726 \mathrm{~cm}^{-1}$ band which confirmed the presence of TPGDA in the polymer matrix. This indicates the successful preparation of the TPGDA-grafted waste rubber via radiation induced grafting techniques.
\end{abstract}

\section{Introduction}

The accumulation of waste rubber into the landfill has posing a serious threat to the health and environment. This is due to their durability and immunity to the biological degradation which makes the disposal and reprocessing of discarded tires is extensively difficult. With an increase in automobile use, the generation of waste rubber from the tire is also increase. In Korea, 28 million tons of scrap tires are produced annually (Ministry of Environment of Korea, 2011) while the United States generates approximately 300 million tons of scrap tires annually [1]. On the other hand, data from Malaysia Industrial Development Authority, MIDA shows that Malaysia generates about 150000 tons of scrap tires every year. These statistics shows the vast number of scrap tires produced annually worldwide. The problems associated with the disposal of the waste rubber are also very clear if not recycle or discarded properly. Increasing concerns regarding to recycling waste rubber and the growing cost of discarded waste to the environment has led to a number of research on the utilization of waste rubber.

*Corresponding author: sitisalwa@nm.gov.my 
However, waste rubber has low mechanical and structural properties which will cause a production of low quality product due to low properties of materials. In order to overcome this disadvantage, the modification techniques by using radiation induced grafting are proposed. Surface modification techniques shows an excellent performance in terms of engineering properties, such as mechanical properties, environmental stability, gas impermeability, flammability as well as adsorption properties $[2,3]$. The grafted product obtained can be tailored into wide range of application especially for industrial application.

To date, there is a lot of research that has been done using radiation-induced grafting to modify the polymer properties such as Uwe Lappan et al. [4] conducted high energy radiation grafting of styrene onto fluoropolymers films and Przybytniak et.al [5] investigated functionalization of polymer surface by radiation-induced grafting of acryclic acid onto polyethylene, polypropylene and polystyrene. In 2014, Nor Azwin Shukri et al. [6] successfully grafted antimicrobial agent onto polyethylene film through radiationinduced graft copolymerization and grafted film showed a strong and stable antibacterial activity. Besides, numbers of study on the utilization of waste rubber from the discarded tires with various area of application have been reported; used as modifiers to asphalt paving mixture and used as an additive to Portland cement concrete in civil engineering application [1], used as a fillers in the composite blending [7] and used in high voltage outdoor insulation application [8]. Although studies could be found, concerning the application of radiation induced grafting to utilize waste rubber. At the author best knowledge, the incorporation of waste rubber with acrylate group n-butyl acrylate, methacrylic acid and tripropylene glycol diacrylate via radiation induced grafting is still scarce in the scientific literature. Moreover, no systematic research has been reported on the effect of grafting condition. Thus, this research is proposed to determine and to compare grafting work of various acrylate groups onto waste rubber. Comparison of these acrylate groups will be a guide for the use of suitable monomer in waste rubber for industrial application.

\section{Methodology}

\subsection{Materials}

Waste rubber from recycle tires used in this study was obtained from Sin Rubtech Consultancy Sdn. Bhd. The monomer used in this study is n-butyl acrylate (n-BA), tripropylene glycol diacrylate (TPGDA) and methacrylic acid (MAA). Solvents and chemical reagents were of laboratory grade and were used without further purification.

\subsection{Radiation-induced grafting}

The waste rubber samples were weighed and immersed in different concentration acrylates namely n-BA, MAA and TPGDA with concentration of $1 \%$ and $10 \%$. The samples were then irradiated using electron beam accelerator operated at voltage of $2 \mathrm{MeV}$ to a dose of 10 $\mathrm{kGy}$ and $100 \mathrm{kGy}$ respectively. The irradiated samples were placed in the water bath at 40 ${ }^{0} \mathrm{C}$ for the grafting reaction to occur. After 30 minutes, the samples were removed from the solution, cleaned by filtration and washed again repeatedly until no subsequent weight decrease. This is to remove the excess monomer and homopolymers. The weight of grafted sample was measured after drying in an oven at $60^{\circ} \mathrm{C}$ until constant weight was achieved.

The grafting yield is defined with the weight basis expression to confirm the grafted copolymer. The grafting percentage was calculated by the percentage weight gain of waste 
rubber after grafting process as defined below (Eq. 1)

$$
G Y(\%)=\left(\left(W_{g}-W_{0}\right) / W_{0}\right) \times 100
$$

Where, $\mathrm{W}_{\mathrm{g}}$ and $\mathrm{W}_{0}$ are denote the weights of grafted and un-grafted waste rubber samples, respectively.

\subsection{Characteristic of waste rubber}

The infrared of grafted waste rubber were obtained using Attenuated Total ReflectanceFourier Transform Infrared Spectroscopy (ATR-FTIR) spectrometer (Mattson 6020-Galaxy Series) in transmittance mode at frequency in the range of $4000-400 \mathrm{~cm}^{-1}$.

\section{Results and discussion}

The effect of absorbed radiation dose (kGy) on the grafting yield (\%) of acrylate monomer onto waste rubber is shown in Figure 1. Grafting yield referred to the amount of monomer used was successfully grafted to the trunk polymer in percentage. In this study, grafting of MAA, n-BA and TPGDA monomer was done using post-irradiation method with doses of $10 \mathrm{kGy}$ and $100 \mathrm{kGy}$. Figure 1 shows the study for radiation induced grating of MAA monomer and n-BA monomer was $-0.99 \%$ and $-1.345 \%$ of grafting yield for $10 \mathrm{kGy}$ $-1.46 \%$ and $-1.575 \%$ of grafting yield for $100 \mathrm{kGy}$ respectively. This clearly shows that there is no grafting occurs for MAA monomer and n-BA monomer since the grafting yield is in negative region for both of the monomer. In contrast, radiation induced grafting of TPGDA monomer for $10 \mathrm{kGy}$ and $100 \mathrm{kGy}$ is $12.285 \%$ and $6.34 \%$ of grafting yield respectively. Only TPGDA monomer was successfully grafted onto waste rubber via radiation induced grafting techniques.

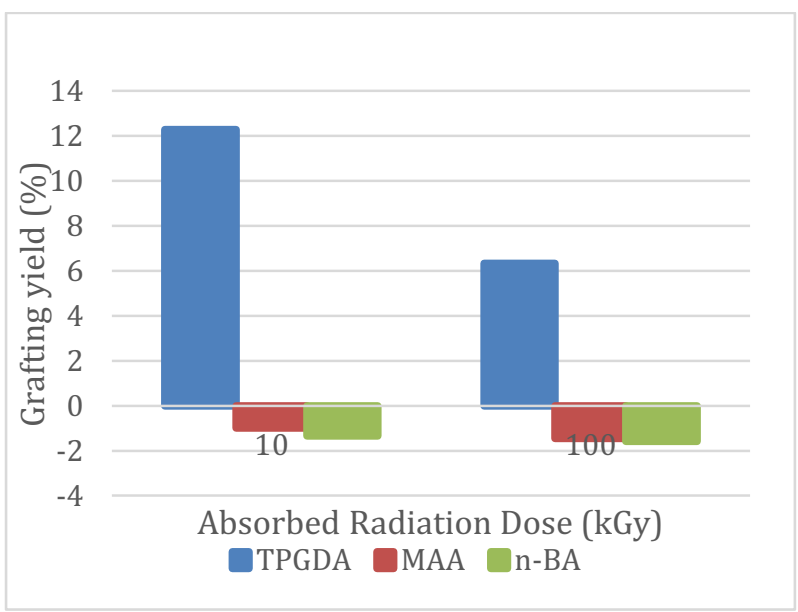

Fig. 1. Graph of absorbed radiation dose (kGy) versus grafting yield (\%)

The effect of monomer concentration (\%) on the grafting yield (\%) of acrylate monomer onto waste rubber is shown in Figure 2. Grafting of MAA, n-BA and TPGDA was done using $1 \%$ and $10 \%$ of monomer concentration. Figure 2 shows the study for radiation induced grating of TPGDA at $1 \%$ concentration is $6.21 \%$ grafting yield. On the 
other hand, MAA and n-BA monomer shows no grafting occurs since both monomer of grafting yield was in negative region. Radiation induced grafting of $10 \%$ monomer concentration is $8.14 \%$ and $8.17 \%$ of grafting yield for TPGDA and MAA monomer respectively. Only TPGDA monomer was successfully grafted onto waste rubber via radiation induced grafting techniques at $1 \%$ and $10 \%$ of monomer concentration.

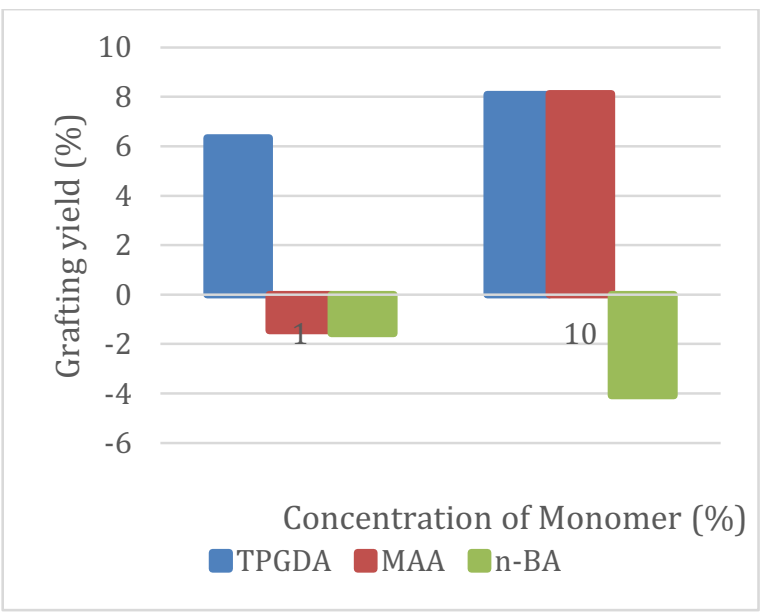

Fig. 2. Graph of concentration of monomer (\%) versus grafting yield (\%)

The radiation induced grafting of TPGDA onto waste rubber was further study with FTIR spectrometer and the FTIR spectrum of the sample is shown in Figure 3. The FTIR spectra shows a new band appear at $1726 \mathrm{~cm}-1$ attributed to $\mathrm{C}=\mathrm{O}$ carbonyl bond from the acrylate group from TPGDA monomer. This band clearly proved the TPGDA monomer was successfully grafted onto the waste rubber via radiation induced grafting.

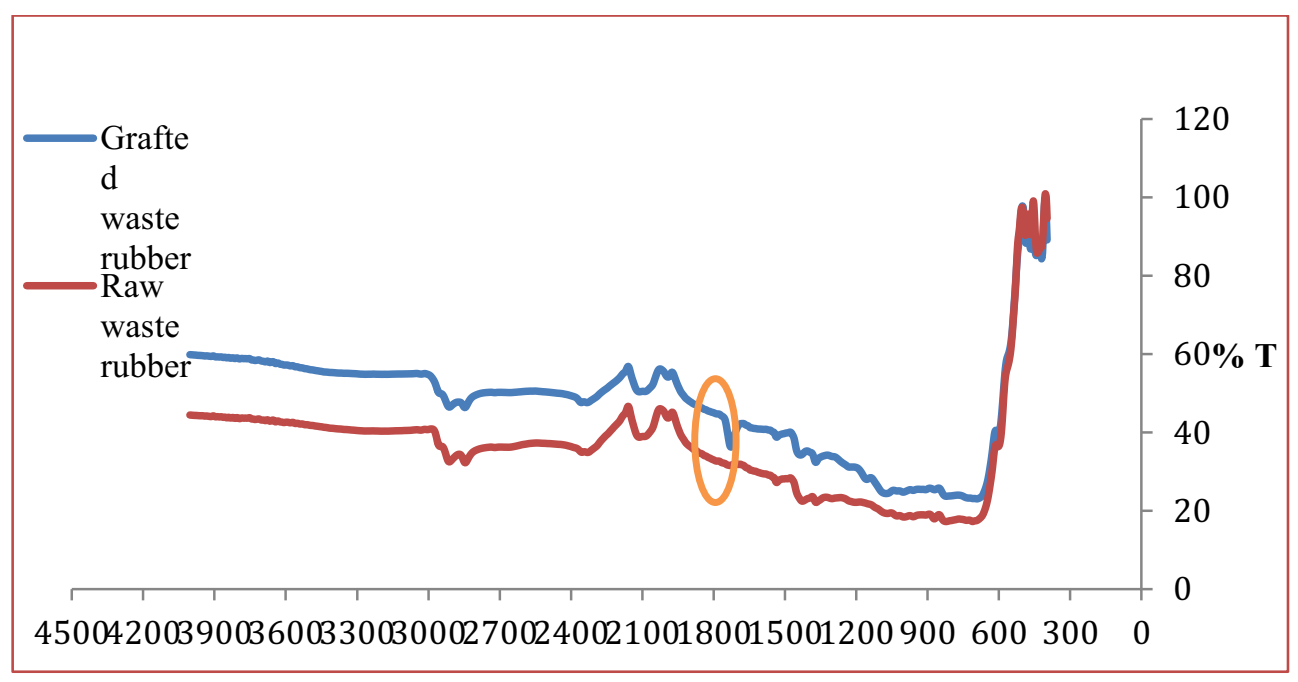

wavenumber $\mathrm{cm}^{-1}$

Fig. 3. FTIR spectra of un-grafted waste rubber and TPGDA grafted waste rubber 


\section{Conclusions}

In conclusion, radiation induced grafting of MAA, n-BA and TPGDA monomer onto waste rubber was studied. Comparing the results of the effect of the absorbed radiation dose and monomer concentration, only TPGDA monomer has shown positive grafting yield. Meanwhile, for MAA and n-BA monomer did not show any grafting yield. This indicates that only TPGDA was successfully grafted onto the surface of waste rubber as proven by FTIR spectrometer. The carbonyl bond attributed to acrylate group from TPGDA monomer was confirmed in the polymer matrix. Thus, TPGDA was chosen as a suitable monomer for this system.

The authors wish to thank to Agensi Nuclear Malaysia and University Malaysia Perlis for the support and guidance throughout this study.

\section{References}

1. Q. Dong, B. Huang, X. Shu, Constr. Build. Mater., 48, 116 (2013)

2. S. Shimada, Y. Takahasi, Y. Sugino, S. Hara, K. Yamamoto, J. Polym. Sci. B. Polym. Phys., 42, 1705 (2004)

3. J.Y. Li, Y.M. Sun, H. Zeng, W.X. Xue, Y. Xiao, Q. Yu, J. Appl. Polym. Sci., 89, 662 (2003)

4. U. Lappan, U. Geibler, U. Gohs, S. Uhlmann, Radiat. Phys. Chem., 79, 1067 (2010)

5. G. Przybytniak, E.M. Kornacka, K. Mirkowski, M. Walo, Z. Zimek, Nukleonika, 53, 89 (2008)

6. S. Nor Azwin, G. Zulkafli, O. Nor Azillah Fatimah, O. Siti Fatahiyah, W. Mat Uzir, Aust J. Basic Appl. Sci, 8, 92 (2014)

7. C.T. Ratnam, S. Ramarad, M. Khalid, Noraini, J. Compos. Biodegradable Polym., 1, 16 (2013)

8. A. Aman, M.M. Yaacob, M.A. Alsaedi, K.A. Ibrahim, Int. J. Elec. Power, 45, 346 (2013) 